\title{
Macromolecular refinement at any resolution using shift field optimization and regularization
}

\author{
Kevin Cowtan, Paul Bond, Scott Hoh \\ University of York, YORK, United Kingdom \\ kevin.cowtan@york.ac.uk
}

For half a century the refinement of atomic model parameters to best explain the observed diffraction pattern has been fundamental to the process of crystallographic structure solution. This process has traditionally been carried out by the optimization of individual atomic parameters, with the use of stereochemical restraints to maintain plausible model geometry, particularly when data resolution is poor. However the data are often too poor to reliably indicate how individual atoms should be moved, and as a result the refinement calculation becomes a protracted battle between the noisy data pulling atoms in different directions and the restraints which are trying to maintain model geometry. This limits both the speed and radius of convergence of the calculation.

Shift field refinement is a new approach in which shifts to the calculated electron density are determined over extended regions of the unit cell, where the region size may be varied according to the resolution of the data and the type of feature (from whole domain to individual atom) being refined. The enables refinement to capture large domain shifts at low resolution, and to be applied at any resolution with rapid convergence. We have already demonstrated improved molecular replacement results when incorporating this step. We now demonstrate how the method can be used to refine a map against a set of diffraction observations, even in the absence of an atomic model.

We also demonstrate how the incorporation of a separate regularization step can be used to improve the refinement results by allowing more cycles of refinement to be run without the risk of model degradation due to accumulated model distortions. This in turn leads to further improvements in the refinement results.

Keywords: refinement, computational methods, macromolecular crystallography 